\title{
Contribuição da concentração total de radionuclídeos aos registros de perfis de raios gama no reservatório Namorado
}

Eara de Souza Luz Oliveira \& Jorge Leonardo Martins, COGE-ON/MCT

Copyright 2010, SBGf - Sociedade Brasileira de Geofísica. Este texto foi preparado para a apresentação no IV Simpósio Brasileiro de Geofísica, Brasília, 14 a 17 de novembro de 2010. Seu conteúdo foi revisado pelo Comitê Técnico do IV SimBGf, mas não necessariamente representa a opinião da SBGf ou de seus associados. É proibida a reprodução total ou parcial deste material para propósitos comerciais sem prévia autorização da SBGf.

\section{RESUMO}

Investigações para fins de estimativa das concentrações de radionuclídeos (i.e., U, Th e K) são realizados em bacias sedimentares utilizando amostras de rochas e/ou perfis geofísicos radioativos. Neste trabalho, propomos a aplicação de um modelo que descreve a variação de raios gama naturais (GR), para avaliar a contribuição da contagem total de radionuclídeos aos perfis de raios gama no reservatório de Namorado, bacia de Campos. Utilizando interpolação pela média ponderada inversa quadrática, construímos mapas com estimativas de contagem total de radionuclídeos (CT) e do calor radiogênico (CR) em profundidades predeterminadas. Os resultados da distribuição de CT e CR nos poços constituem os pontos de controle para a estimativa de tais mapas. Os resultados aqui obtidos serão utilizados em um processo inverso, a saber, na decomposição de CT em mapas de concentrações de radionuclídeos.

\section{INTRODUÇÃO}

As concentrações de radionuclídeos naturais (U, Th e K) presentes nas rochas crustais são normalmente estimadas a partir de amostras de rochas tratadas em laboratórios, uma prática necessária para estudos da litologia local. Isso porque os minerais possuem concentrações proporcionais de radionuclídeos naturais, e, devido ao processo de decaimento radioativo, emitem radiações que podem ser registradas por detectores de raios gama, também presentes em ferramentas de perfilagem geofísica nuclear (perfis de raios gama). Paralelamente a emanação de radioatividade gama natural, ocorre a produção de calor que atua na formação de hidrocarbonetos através do processo conhecido por maturação térmica de kerogênio - vide (Roque and Ribeiro, 1997). Alguns trabalhos tem sido realizados em diferentes bacias sedimentares para estimativa das concentrações de radionucídeos (Conceição and Bonotto, 2006; Ferreira, 2008). Outros trabalhos, também em bacias sedimentares, avaliaram a taxa de produção de calor radiogênico na área de estudo (Sapucaia et al., 2005). Além dos conhecimentos produzidos nesses trabalhos especificamente para bacias sedimentares, a estimativa das concentrações de radionuclídeos e da produção de calor radiogênico constituem informações complementares para os estudos do fluxo geotérmico. No entanto, nem sempre é possível a coleta de amostras de rochas para a determinação dessas quantidades físicas.

Utilizando a relação linear entre a produção de calor radiogênico e a contagem de raios gama naturais apresentada em Bucker \& Rybach (1996), este trabalho visa o desenvolvimento de uma metodologia para estimativa das concentrações de radionuclídeo e produção de calor radiogênico no reservatório de Namorado, Bacia de Campos, a partir dos registros de perfis de raios gama. Paralelamente, estudamos a descrição faciológica do perfil litológico correspondente e os valores de produção de calor radiogênico e contagem total de radionuclídeos. $O$ reservatório em estudo, de origem turbidítica, está inserido na formação Macaé superior. As litologias predominantes nessa formação são arenitos e folhelhos, respectivamente com a matriz quartzoza e k-feldspática. Por conseguinte, esperamos a ocorrência de concentrações de potássio significativamente maiores que as normais.

\section{METODOLOGIA}

A formulação do modelo físico que descreve a variação das leituras nos perfis de raios gama naturais em função das contagens de radionuclídeos utiliza a equação de produção de calor radiogênico apresentada em Bucker \& Rybach (1996), a saber,

$$
A=10^{-5} \rho\left(9.52 C_{U}+2.56 C_{T h}+3.48 C_{K}\right),
$$

onde $A$, em $\mu \mathrm{W} / \mathrm{m}^{3}$, representa o calor radiogênico, $\rho$ é a densidade da rocha, em $\mathrm{Kg} / \mathrm{m}^{3}$. Os parâmetros $C_{U}$ e $C_{T h}$, em ppm, e $C_{K}$, em \%, representam as concentrações de urânio, tório e potássio, respectivamente.

A partir das informações de análise de testemunhos dos poços do reservatório de Namorado, derivamos a seguinte relação linear entre a produção de calor radiogênico e o registro da contagem de raios gama naturais,

$$
A=2.0 \times 10^{-2} \mathrm{GR}+2.2,
$$

onde os registros de GR são expressos em unidades API. Igualando as equações (1) e (2), podemos derivar facilmente o modelo físico $\mathrm{GR} \equiv \mathrm{GR}\left(\rho, C_{U}, C_{T h}, C_{k}\right)$, em unidades API, como segue:

$$
\mathrm{GR}=\rho\left(a_{u} C_{U}+a_{t h} C_{T h}+a_{k} C_{K}\right)+a_{g r} .
$$

A contagem total de radionuclídeos (CT) representa a contribuição das concentrações de $\mathrm{U}$, Th e K, respectivamente, às leituras do perfil de raios gama, sendo definida 


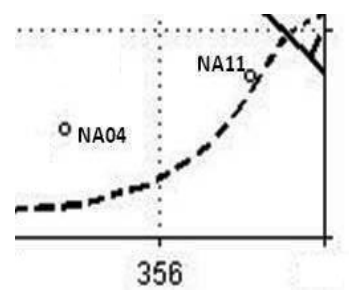

Figura 1: Mapa estrutural parcial do reservatório de Namorado, exibindo apenas a localização dos poços NA04 e NA11. Limites do reservatório: linha pontilhada, representando contato não inferido e, no canto superior direito, um trecho de uma falha normal. As coordenadas UTM estão em km.

por:

$$
\mathrm{CT}=\left(a_{u} C_{U}+a_{t h} C_{T h}+a_{k} C_{K}\right) .
$$

Assim, escrevemos

$$
\mathrm{GR}=\rho \mathrm{CT}-a_{g r},
$$

onde as constantes $a_{u}, a_{t h}\left(\mathrm{~m}^{3} / \mathrm{Kg} / \mathrm{ppm}\right)$ e $a_{k}\left(\mathrm{~m}^{3} / \mathrm{Kg} / \%\right)$ assumem valores de acordo com a relação linear expressa em (2), enquanto $a_{g r}=1.1 \times 10^{2}$ unidades API.

Aplicamos as equações 2 e 4 aos poços NA04 e NA11, para fins de avaliar a variação em profundidade da contribuição total de radionuclídeos e da produço de calor radioênico a partir do perfil GR. Aplicamos em 39 poços do reservatório de Namorado - profundidades entre 2950 $\mathrm{m}$ e $3150 \mathrm{~m}$. Construímos mapas da variação dessas quantidades físicas para três profundidades $(3000 \mathrm{~m}$, $3040 \mathrm{~m}$ e $3090 \mathrm{~m}$ ). Na construção desses mapas, utilizamos um interpolador tipo média ponderada inversa quadrática proposto em Weber \& Englund (1992) e Weber \& Englund (1994). Para efeito de comparação, analisamos as descrições faciológicas das litologias disponíveis nas profundidades citadas acima de dois poços adjacentes, designados NA04 e NA11 na figura 1. Na tabela (1) estão apresentadas as descrições faciológicas das litologias correspondentes encontradas nas profundidades consideradas no presente trabalho.

Tabela 1: Descrição da litologia; Z é a profundidade em metros.

\begin{tabular}{ccc}
\hline$Z$ & NA04 & NA11 \\
\hline 3000 & Não informado & Não informado \\
3040 & Arenito médio & Marga bioturbada \\
3090 & Arenito médio & Interlaminado lamoso
\end{tabular}

\section{RESULTADOS}

Nas figuras 2 e 3, observamos que tais variações acompanham as mesmas anomalias apresentadas pelo perfil

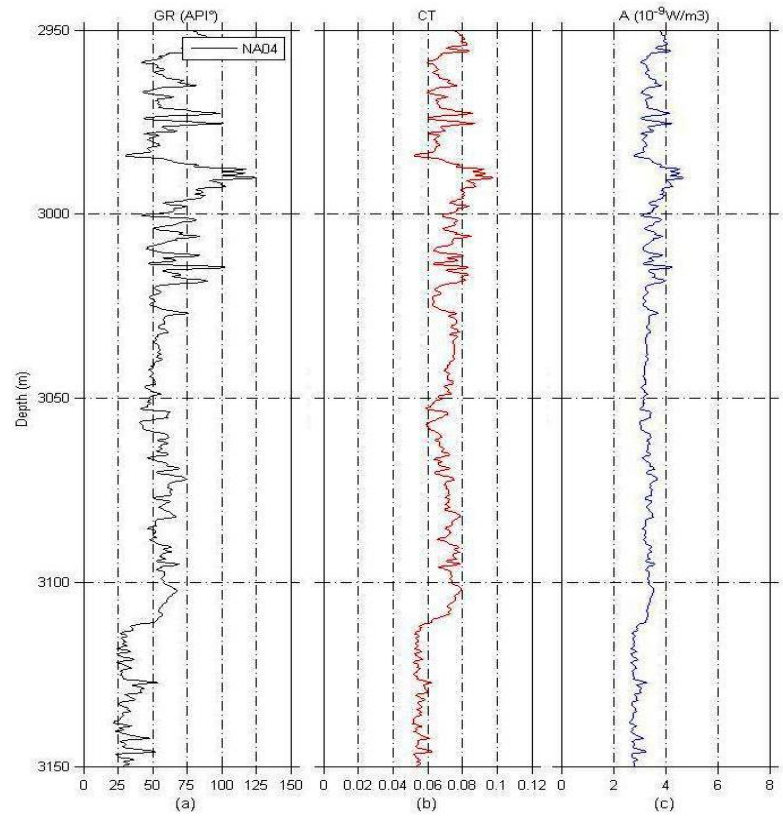

Figura 2: Perfis geofísicos do poço NA04: (a) perfil de raios gama (unidades $\mathrm{API}$ ); (b) perfil de contagem total de radionuclídeos (unidades $A P I \times \mathrm{m}^{3} / \mathrm{Kg}$,); e (c) perfil de calor radiogênico $\left(\mu \mathrm{W} / \mathrm{m}^{3}\right)$.

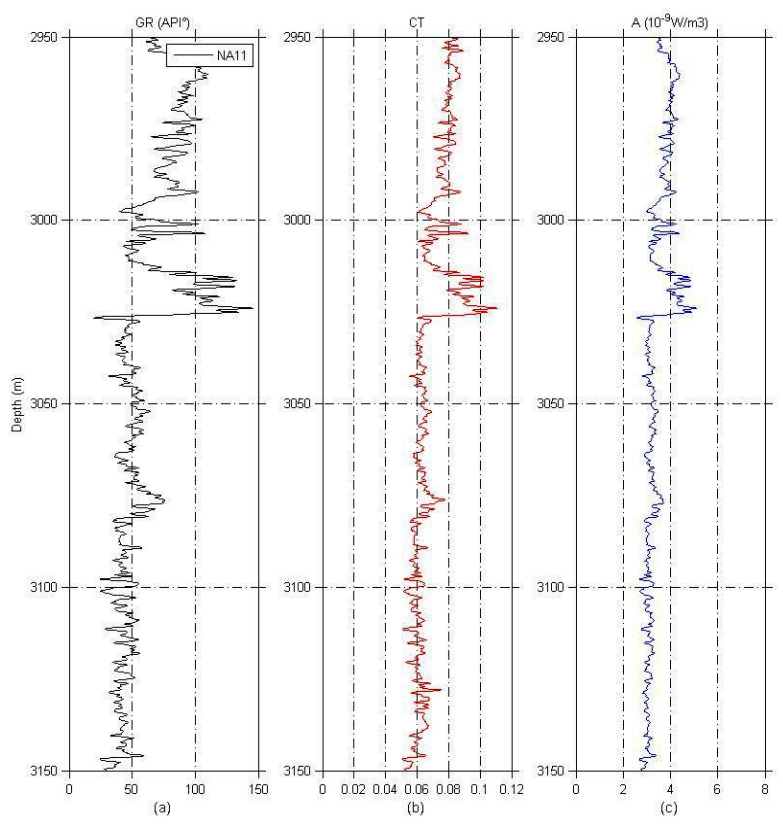

Figura 3: Perfis geofísicos do poço NA11: (a) perfil de raios gama (unidades $\mathrm{API}$ ); (b) perfil de contagem total de radionuclídeos (unidades $A P I \times \mathrm{m}^{3} / \mathrm{Kg}$,); e (c) perfil de calor radiogênico $\left(\mu \mathrm{W} / \mathrm{m}^{3}\right)$.

GR. De fato, esse é um resultado esperado, pois, segundo os modelos nas equações 2 e 4, CT e A correspondem a duas quantidades influenciadas pela radioa- 


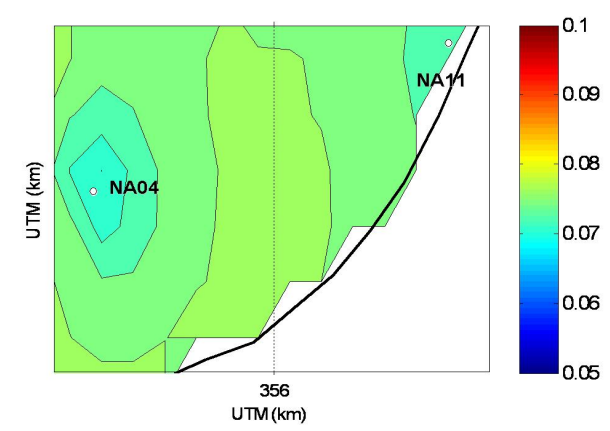

Figura 4: Mapa da contagem total de radionuclídeos (unidades $\mathrm{API} \times \mathrm{m}^{3} / \mathrm{Kg}$ ) na profundidade de $3000 \mathrm{~m}$.

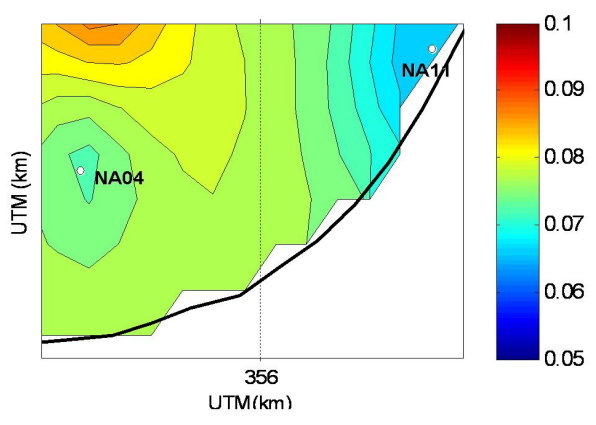

Figura 5: Mapa da contagem total de radionuclídeos (unidades API $\times \mathrm{m}^{3} / \mathrm{Kg}$ ) na profundidade de $3040 \mathrm{~m}$.

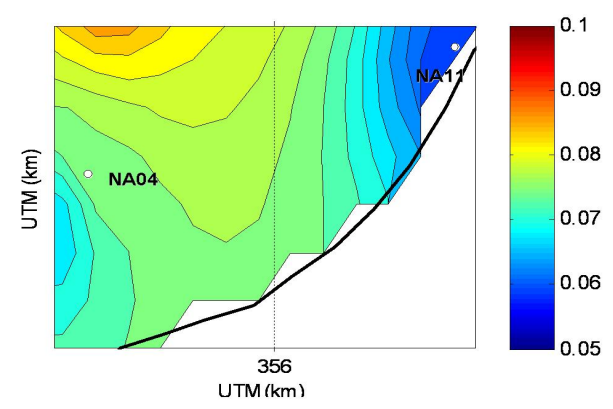

Figura 6: Mapa da contagem total de radionuclídeos (unidades $\mathrm{API} \times \mathrm{m}^{3} / \mathrm{Kg}$ ) na profundidade de $3090 \mathrm{~m}$.

tividade natural gama.

Adicionalmente, construímos mapas representativos das variações espaciais de CT (figuras 4,5 e 6 ) e de A (figuras 7, 8 e 9) nas profundidades de $3000 \mathrm{~m}, 3040 \mathrm{~m} \mathrm{e}$ $3090 \mathrm{~m}$.

Notamos que, para os poços NA04 e NA11, tanto em profundidade como espacialmente (entre os poços NA04 e NA11), as magnitudes de CT a de A são pequenas e aproximadamente iguais. Analisando os mapas de CT, observamos diferentes feições. Entre 3000 m e 3040 m: (1) ocorre uma estrutura geológica acima do poço NA04; (2) ocorre decréscimo de CT (valores entre 0.07 e 0.08 unidades API $\times \mathrm{m}^{3} / \mathrm{Kg}$ para 0.06 e 0.07 unidades API

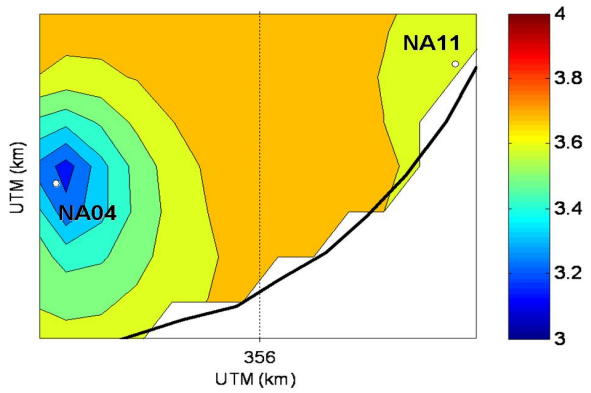

Figura 7: Mapa de calor radiogênico $\left(\mu \mathrm{W} / \mathrm{m}^{3}\right)$ na profundidade de $3000 \mathrm{~m}$.

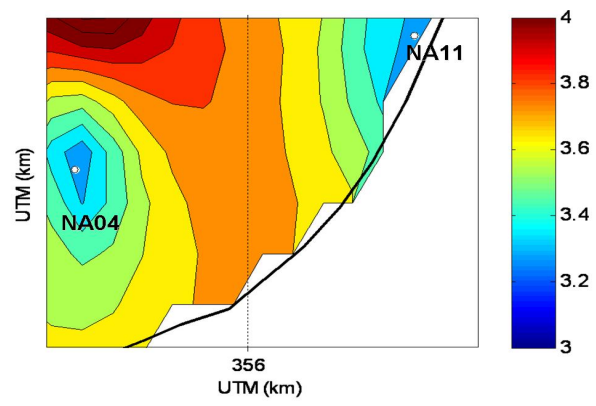

Figura 8: Mapa de calor radiogênico $\left(\mu \mathrm{W} / \mathrm{m}^{3}\right)$ na profundidade de $3040 \mathrm{~m}$.

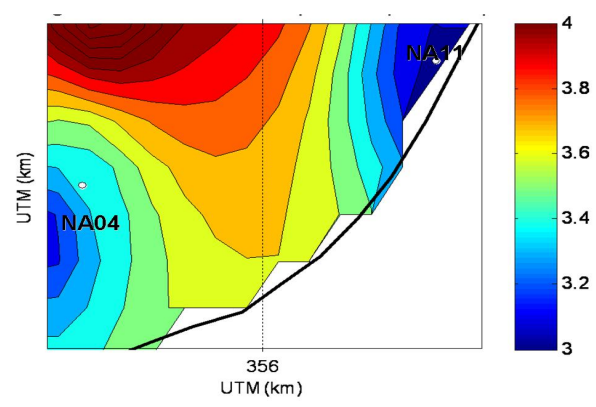

Figura 9: Mapa de calor radiogênico $\left(\mu \mathrm{W} / \mathrm{m}^{3}\right)$ na profundidade de $3090 \mathrm{~m}$.

$\times \mathrm{m}^{3} / \mathrm{Kg}$ ) nas imediações do poço NA11, e (3) ocorrem variações significativas de CT entre esse poços, indicando a continuidade de estruturas geológicas. Por outro lado, entre 3040 m e 3090 m: (1) a estrutura geológica acima do poço NA04 mantém a sua influência na variação de $\mathrm{CT}$; (2) a anomalia antes presente nas imediações de NA04, se desloca levemente para a esquerda; (3) as anomalias nas imediações do poço NA11 sofrem um ligeiro decréscimo para a esquerda, e (4) as anomalias entre os poços permanecem no entanto, os valores de CT no poço NA11 sofre outro decréscimo do intervalo 0.06-0.07 unidades API $\times \mathrm{m}^{3} / \mathrm{Kg}$ para 0.05 0.06 unidades API $\times \mathrm{m}^{3} / \mathrm{Kg}$. Em termos gerais, podemos aplicar as mesmas considerações aos mapas de variação da produção de calor radiogênico com valores 
continuos entre 3.0 e $4.0 \mu \mathrm{W} / \mathrm{m}^{3}$, figuras 7,8 e 9 .

\section{DISCUSSÃO E CONCLUSÕES}

A relação linear usada para calcular o calor radiogênico apresenta uma resposta positiva para os poços do reservatório Namorado. Este resultado possibilita a aplicação da relação em estudos de investigação de concentrações de radionuclídeos no reservatório em estudo. Pode-se observar a linearidade da relação em reservatórios sedimentares, porém há uma particularidade nas constantes para cada reservatório que está, provavelmente, diretamente relacionado ao tipo de formação sedimentar do reservatório. Portanto, é necessário investigar as condições litológicas dos poços para uma correta linearização. Quanto as informações básicas para a estimativa das concentrações de radionuclídeos naturais, tanto a produção de calor radiogênico quanto a Contagem Total são bons índices. A baixa resolução dos resultados pode dificultar a caracterizaão sedimentar do reservatório no entanto, por serem funções das concentrações, informações complementares serão essenciais para o objetivo que ser desejar alcançar. Uma investigação futura em outros poços do mesmo reservaório poderá confirmar as expectativas aqui geradas. Aliado às informações da formação litológica do reservatório local os valores de Contagem Total e Produção de Calor Radiogênico serão utilizados para estudos da estimativa das concentrações de urânio, tório e potássio.

\section{AGRADECIMENTOS}

Eara Luz agradece ao auxílio financeiro oferecido pela CAPES para o desenvolvimento de tese de doutorado no Curso de PG em Geofísica no Observatório Nacional.

\section{REFERÊNCIAS}

Bucker, C., and Rybach, L., 1996, A simple method to determine heat production from gamma-ray logs.: Elsevier Science Ltd, 3, no. 4, 373-375.

Conceição, F. T., and Bonotto, D. M., 2006, Dose de exposição radiométrica e composição das rochas sedimentares e ígneas na Bacia do rio Corumbataí: Rev. Bras. Geof., 24, 37-48.

Ferreira, A. O., 2008, Distribuição de radionuclídeos em um afloramento de sedimentos permianos da formação Irataí na Bacia do Paraná: Universidade Estadual de Londrina.

Roque, A., and Ribeiro, F. B., 1997, Radioactivity and Radiogenic Heat Production in the Sediments of the São Francisco Sedimentary Basin, Central Brazil: Elsevier Science Ltd, 48, no. 3, 413-422.

Sapucaia, N. S., Argollo, R., and Barbosa, J. S. F., 2005, Teores de potássio, urânio, tório e taxa de produção de calor radiogênico no embasamento adjacente às bacias sedimentares de Camamu e Almada, Bahia, Brasil.: Rev. Bras. Geof., pages 435-475.
Weber, D. D., and Englund, E. J., 1992, Evaluation and comparison of spatial interpolators:

Weber, D. D., and Englund, E. J., 1994, Evaluation and comparison of spatial interpolators II: 